\title{
The Origin and Evolution of Cosmic Magnetism
}

\author{
B. M. Gaensler ${ }^{\mathrm{a}}$, R. Beck ${ }^{\mathrm{b}}$ and L. Feretti ${ }^{\mathrm{c}}$ \\ ${ }^{a}$ Harvard-Smithonian Center for Astrophysics, 60 Garden Street MS-6, Cambridge, MA 02138, USA \\ bMax-Planck-Institut für Radioastronomie, Auf dem Hügel 69, 53121 Bonn, Germany \\ ${ }^{\mathrm{c}}$ Istituto di Radioastronomia CNR/INAF, Via Gobetti 101, 40129 Bologna, Italy
}

\begin{abstract}
Magnetism is one of the four fundamental forces. However, the origin of magnetic fields in stars, galaxies and clusters is an open problem in astrophysics and fundamental physics. When and how were the first fields generated? Are present-day magnetic fields a result of dynamo action, or do they represent persistent primordial magnetism? What role do magnetic fields play in turbulence, cosmic ray acceleration and galaxy formation? Here we demonstrate how the Square Kilometer Array (SKA) can deliver new data which will directly address these currently unanswered issues. Much of what we present is based on an all-sky survey of rotation measures, in which Faraday rotation towards $>10^{7}$ background sources will provide a dense grid for probing magnetism in the Milky Way, in nearby galaxies, and in distant galaxies, clusters and protogalaxies. Using these data, we can map out the evolution of magnetised structures from redshifts $z>3$ to the present, can distinguish between different origins for seed magnetic fields in galaxies, and can develop a detailed model of the magnetic field geometry of the intergalactic medium and of the overall Universe. With the unprecedented capabilities of the SKA, the window to the Magnetic Universe can finally be opened.
\end{abstract}

\section{Introduction}

Understanding the Universe is impossible without understanding magnetic fields. They fill intracluster and interstellar space, affect the evolution of galaxies and galaxy clusters, contribute significantly to the total pressure of interstellar gas, are essential for the onset of star formation, and control the density and distribution of cosmic rays in the interstellar medium (ISM). But in spite of their importance, the evolution, structure and origin of magnetic fields are all still open problems in fundamental physics and astrophysics. Specifically, we still do not know how magnetic fields are generated and maintained, how magnetic fields evolve as galaxies evolve, what the strength and structure of the magnetic field of the intergalactic medium (IGM) might be, or whether fields in galaxies and clusters are primordial or generated at later epochs. Ultimately, we would like to establish whether there is a connection between magnetic field formation and structure formation in the early Universe, and to obtain constraints on when and how the first mag- netic fields in the Universe were generated.

Most of what we know about astrophysical magnetic fields comes through the detection of radio waves. Synchrotron emission measures the total field strength, while its polarization yields the orientation of the regular field in the sky plane and also gives the field's degree of ordering (see Figure (1). Incorporating Faraday rotation yields a full three-dimensional view by providing information on the field component along the line of sight (see Figure 2), while the Zeeman effect provides an independent measure of field strength in cold gas clouds. However, measuring astrophysical magnetic fields is a difficult topic still in its infancy, restricted to nearby or bright objects. In this Chapter we explain how the Square Kilometre Array (SKA) can revolutionise the study of Cosmic Magnetism.

\section{Polarimetry with the SKA}

Much of what the SKA can contribute to our understanding of magnetic fields will come from its polarimetric capabilities. As discussed in more 


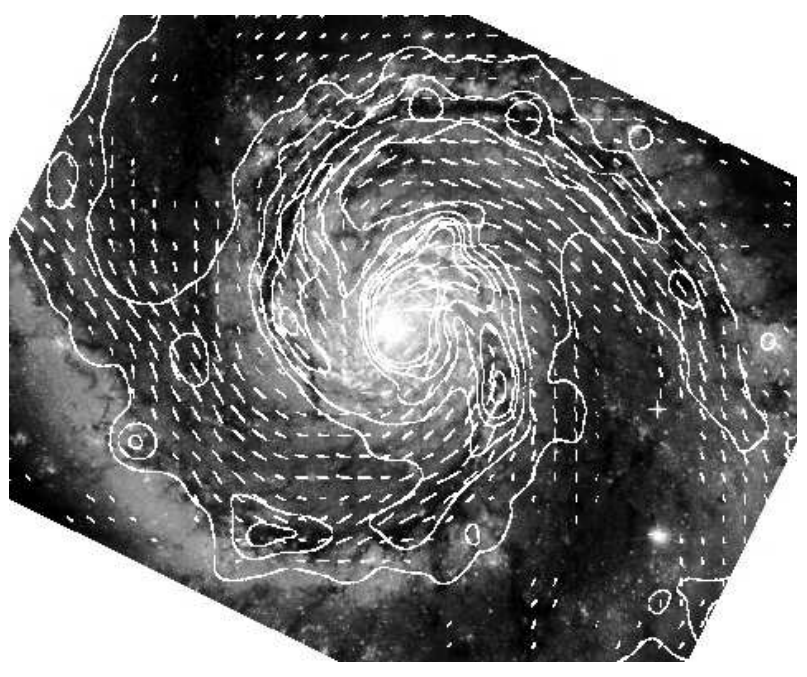

Figure 1. The magnetic field of the grand design spiral galaxy M 51. The image shows an optical HST image, overlaid with contours showing the radio total intensity emission at $5 \mathrm{GHz}$. The vectors show the orientation of the magnetic field, as determined from $5 \mathrm{GHz}$ linear polarization measurements (Faraday rotation is small at this frequency) [13]. detail elsewhere 312 , the main SKA specifications which enable this capability will be high polarization purity and spectropolarimetric capability. The former will allow detection of the relatively low linearly polarized fractions ( $\lesssim 1 \%$ ) from most astrophysical sources seen down to sub-mJy levels, while the latter will enable accurate measurements of Faraday rotation measures (RMs), intrinsic polarization position angles and Zeeman splitting. Other minimum requirements include a frequency coverage of 0.5 $10 \mathrm{GHz}$, a field of view at $1.4 \mathrm{GHz}$ of $1 \mathrm{deg}^{2}$ which can be fully imaged at $1^{\prime \prime}$ resolution, a significant $(\sim 50 \%)$ concentration of the collecting area into a central core of diameter $\sim 5 \mathrm{~km}$, and a longest baseline of $\sim 300 \mathrm{~km}$.

It is important to appreciate how powerful the combination of high sensitivity, good polarization purity and full spectropolarimetry of the SKA will be. With current instruments, the only way to simultaneously determine accurate values for both the RM and intrinsic polarization position angle is to make many observations across a broad frequency range. This is not only timeconsuming, but the analysis must proceed cautiously, since various depolarizing effects have a strong frequency dependence (e.g., 36]).

The high sensitivity and broad bandwidth of the SKA eliminate these difficulties: a single spectropolarimetric observation at a single IF can simultaneously provide good estimates of both RM and position angle, the limiting factor often only being the accuracy of ionospheric corrections to the observed Faraday rotation. For example, at an observing frequency of $1.4 \mathrm{GHz}$ with a fractional bandwidth of $25 \%$, a 1-min SKA observation of a source with a linearly polarized surface brightness of $\sim 8 \mu \mathrm{Jy}^{\text {beam }^{-1}}$ will yield a $\mathrm{RM}$ determined to an accuracy $\Delta \mathrm{RM} \approx \pm 5 \mathrm{rad} \mathrm{m}^{-2}$, and its intrinsic position angle measured to within $\Delta \Theta \approx \pm 10^{\circ}$. Measurements of this precision will be routinely available in virtually any SKA observation of a polarized source.

\section{All-Sky Rotation Measures}

Currently $\sim 1200$ extragalactic sources and $\sim 300$ pulsars have measured RMs. These data 


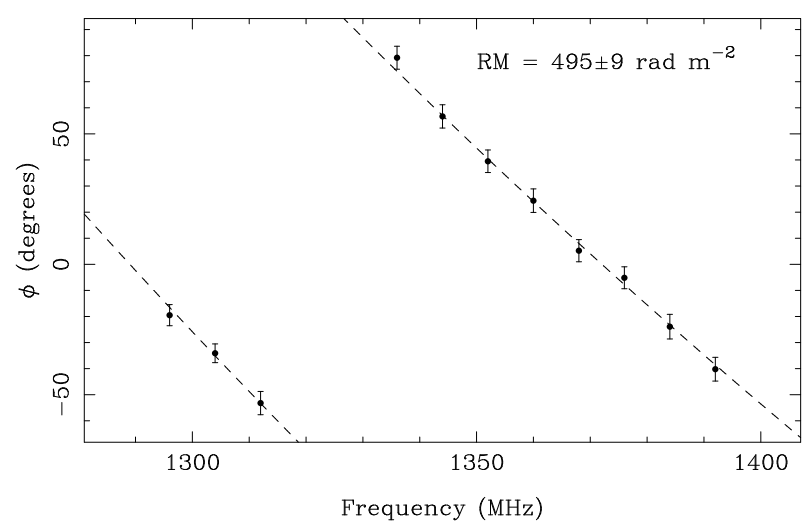

Figure 2. Spectropolarimetry of pulsar B1154-62, demonstrating how the RM can be accurately determined using the position angle of polarization in multiple channels within the same observing band 18.

have proved useful probes of magnetic fields in the Milky Way, in nearby galaxies, in clusters, and in distant Ly $\alpha$ absorbers. However, the sampling of such measurements over the sky is very sparse, and most measurements are at high Galactic latitudes.

A key platform on which to base the SKA's studies of cosmic magnetism will be to carry out an All-Sky RM Survey, in which spectropolarimetric continuum imaging of $10000 \mathrm{deg}^{2}$ of the sky can yield RMs for approximately $2 \times 10^{4}$ pulsars and $2 \times 10^{7}$ compact polarized extragalactic sources in about a year of observing time (see 3] for a detailed description). This data set will provide a grid of RMs at a mean spacing of $\sim 30^{\prime}$ between pulsars and just $\sim 90^{\prime \prime}$ between extragalactic sources (see Figure 3).

In the following Sections, we describe some of the SKA experiments which will provide new insight into the origin, structure and evolution of cosmic magnetic fields. Many of these observations stem directly from the RM grid just described, or from deeper observations of specific regions which will provide an even more closely spaced set of background RMs. This discussion

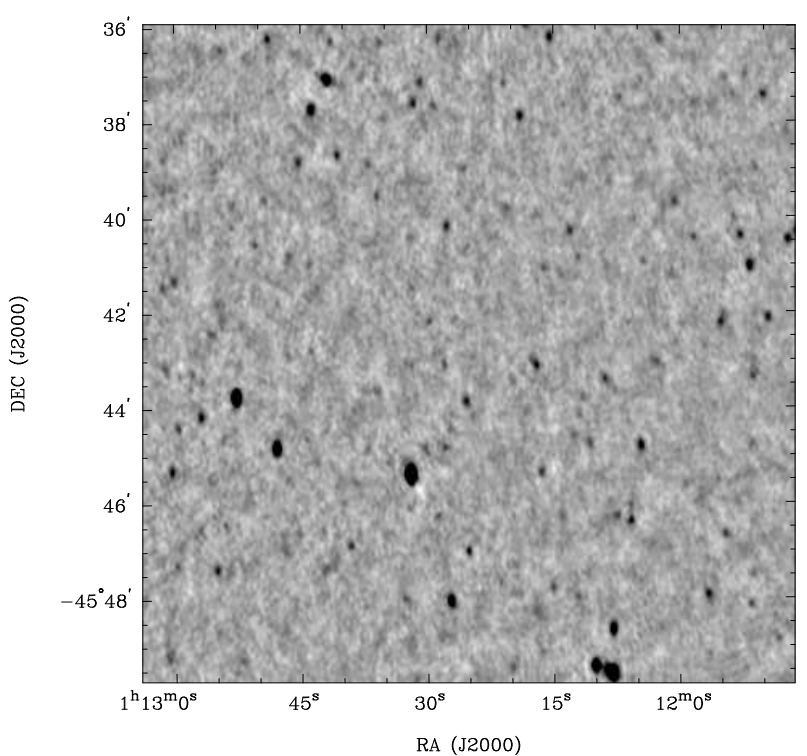

Figure 3. $1.4 \mathrm{GHz}$ ATCA image of a small section of the Phoenix Deep Field 24. The density of extragalactic sources in this total intensity image is similar to what will be achievable in polarized intensity in a 1-hour $1.4 \mathrm{GHz}$ observation with the SKA. 
begins with the Milky Way and its ISM, and then considers other sources at increasingly larger distances.

\section{The Local Universe}

\subsection{The Milky Way and Nearby Galaxies}

Key information on the processes which amplify and sustain large-scale magnetic field structure in galaxies comes from characterising and comparing the three-dimensional structure of the magnetic field in nearby galaxies. Amongst the questions which we would like to resolve are the number and location of magnetic reversals, the relation between magnetic and optical spiral arms, the vertical magnetic structure in the disk, the strength and structure of magnetic fields in galactic halos, and the correspondence between magnetic field structure and other parameters such as rotation speed, Hubble type and star formation rate.

In our Milky Way, the large sample of pulsar RMs obtained with the SKA, combined with distance estimates to these sources from parallax or from their dispersion measures, can be inverted to yield a complete delineation of the magnetic field in the spiral arms and disk on scales $\gtrsim 100$ pc (e.g., 37]). Small-scale structure and turbulence can be probed using Faraday tomography, in which foreground ionised gas produces complicated frequency dependent Faraday features when viewed against diffuse Galactic polarized radio emission (e.g., [172339]). Magnetic field geometries in the Galactic halo and outer parts of the disk can be studied using the extragalactic RM grid (see detailed discussion by [3]).

In external galaxies, magnetic fields can be directly traced by diffuse synchrotron emission and its polarization. For the nearest galaxies, the SKA will provide sufficient resolution and sensitivity to carry out Faraday tomography on this emission, allowing us to identify individual features, structures and turbulent processes in the magnetoionised ISM of these galaxies. These measurements can be compared with $\mathrm{HI}, \mathrm{H} \alpha$ and continuum observations at a variety of wavelengths to establish, in a much more direct way than is possible for the confused lines of sight of our own Galaxy, how magnetic features interact with other features in the ISM.

Synchrotron emission is seen only in magnetised regions in which either cosmic ray electrons have been accelerated, or into which such electrons have diffused. In other parts of a galaxy, particularly in the outer disk and halo, we must rely on background RMs to map magnetic fields. However, the current density of such sources is such that only a handful of nearby galaxies can be studied in this way (e.g., 22]). With the sensitivity of the SKA, deep observations of nearby galaxies can provide $>10^{5}$ background RMs, and thus allow fantastically detailed maps of the magnetic structure. Even at a distance of $10 \mathrm{Mpc}$, most galaxies should be traversed by $\sim 50$ sightlines to polarized background sources. The sample of galaxies which could be studied in this way is very large.

As discussed in detail by [3], the overall structure of galactic magnetic fields provides a potential discriminant between dynamo and primordial mechanisms for the origin and sustainment of these fields 5. For example, the mean-field $\alpha-$ $\Omega$ dynamo model makes specific predictions for the symmetries seen in both azimuthal and vertical field structure. Observations of background RMs and of diffuse polarized synchrotron emission can allow direct measurements of the azimuthal modes, but these measurements are limited at present to $\sim 20$ galaxies [2]. With the SKA, this sample can be increased by up to three orders of magnitude. These data can allow us to distinguish between different conditions (e.g., strong density waves, high star formation rate, or interactions) for excitation of various dynamo modes. Meanwhile, the presence and prevalence of reversals in the disk field structure, plus the structure of field in the halo, will together let us distinguish between dynamo and primordial models for field origin in different systems.

\subsection{Galaxy Clusters}

In clusters of galaxies, magnetic fields play a critical role in regulating heat conduction [1030, and may also both govern and trace cluster formation and evolution. Estimates of the overall magnetic field strength come from inverse Comp- 
ton detections in X-rays, from detection of diffuse synchrotron emission, from cold fronts and from simulations, but our only direct measurements of field strengths and geometries come from RMs of background sources (see [12). Currently just 1-5 such RM measurements can be made per cluster (e.g., 20]); only by considering an ensemble of RMs averaged over many systems can a crude picture of cluster magnetic field structures be established 11.

With the SKA, the RM grid can provide $\sim 1000$ background RMs behind a typical nearby cluster; a comparable number of RMs can be obtained for a more distant cluster through a deep targeted observation. These data will allow us to derive a detailed map of the field in each cluster. With such information, careful comparisons of the field properties in various types of cluster (e.g., those containing cooling flows, those showing recent merger activity, etc.) at various distances can be easily made. Furthermore, detailed comparisons between RM distributions and X-ray images of clusters will become possible, allowing us to relate the efficiency of thermal conduction to the magnetic properties of different regions, and to directly study the interplay between magnetic fields and hot gas.

Deep observations targeting individual clusters will also allow the detection of low surface brightness synchrotron emission, allowing us to explore the role of low-level magnetic fields, and providing vital clues to the evolution of cluster magnetic fields.

\section{Galaxies at Intermediate Redshifts}

Many galaxies at intermediate redshifts $(0.1 \lesssim$ $z \lesssim 2$ ) are representative of the local population but at earlier epochs. Measurements of the magnetic field in such systems thus provides direct information on how magnetised structures evolve and amplify as galaxies mature. However, the linearly polarized emission from galaxies at these distances will often be too faint to detect directly; Faraday rotation thus holds the key to studying magnetism in these distant sources.

\subsection{Galaxies in the Foreground of Bright Extended Radio Sources}

At intermediate redshifts, we expect galaxies to be $<1^{\prime}$ in extent, too small to be adequately probed by the RM grid of compact background sources discussed in 3 However, there are many distant extended polarized sources (e.g., many of the quasars and radio galaxies in the $3 \mathrm{C}$ catalogue), which provide ideal background illumination for probing Faraday rotation in galaxies which happen to lie along the same line of sight. These experiments can deliver maps of magnetic field structures in galaxies more than 100 times more distant than discussed in 4.1 above, and thus provide information on the evolution of magnetic fields as a function of cosmic epoch. Currently this technique has been applicable in just a few fortuitous cases, such as for NGC 1310 seen projected against a lobe of Fornax A (Figure 4 [1435]), and for an absorption line line system at $z=0.395$ seen against PKS 1229-021 29. With the greater sensitivity of the SKA, many such systems lying in front of bright polarized radio sources can be targeted. These objects are less evolved counterparts to the nearby galaxies discussed in 4.1 comparison of the two populations will demonstrate how present day magnetic fields emerge. For example, the $z=0.395$ system discussed by Kronberg et al. 29] appears to be a spiral galaxy with a bisymmetric magnetic field geometry and a magnetic field strength of $\sim 1-4 \mu \mathrm{G}$, surprisingly similar to results from nearby galaxies. Clearly a significant expansion of the sample of such systems can provide strong constraints on the processes which generate magnetic fields in galaxies.

\subsection{RM Statistics of $\mathrm{Ly} \alpha$ Absorbers}

While the approach in $\$ 5.1$ above allows us to study a variety of individual systems in detail, an even larger sample, suitable for statistical studies, can be accumulated by considering unresolved sources in the foreground of our RM grid. Specifically, we expect that a large number of the sources for which we measure RMs will be quasars showing foreground Ly $\alpha$ absorption; these absorption systems likely represent the progenitors of present-day galaxies. If a large enough 


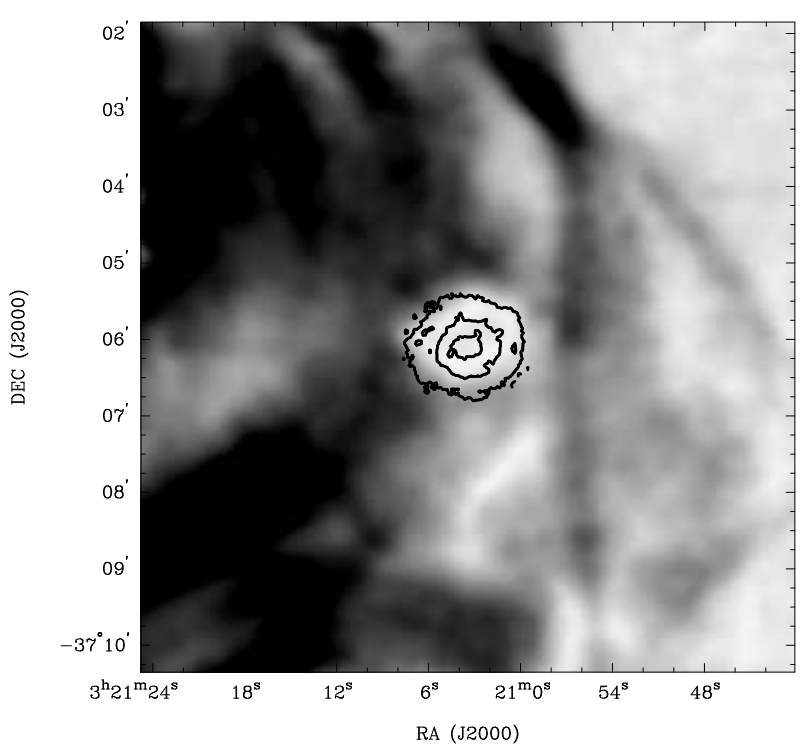

Figure 4. A 1.4 GHz VLA image of linearly polarized intensity from a small region in the western lobe of Fornax A, superimposed with optical contours from the Digitized Sky Survey. The bright optical source corresponds to the spiral galaxy NGC 1310, which produces strong depolarization through its Faraday rotation towards the background polarized emission from Fornax A [14. sample of RMs for quasars at known redshift can be accumulated, a trend of RM vs $z$ can potentially be identified. The form of this trend can then be used to distinguish between RMs resulting from magnetic fields in the quasars themselves and those produced by fields in foreground absorbing clouds (e.g., [42]); detection of the latter effect would then directly trace the evolution of magnetic field in galaxies and their progenitors. This experiment has been attempted several times with existing data sets, but only a relatively small number of absorbing systems show a clear excess in RM. These data correspondingly provide only marginal (if any) evidence for any evolution of RM with redshift 42443231 . There are several limitations in these studies, all of which can be circumvented using the SKA.

First, all quasar RM measurements are contaminated by the Galactic contribution to the RM. The Galactic signal can be removed by considering the RMs of neighboring background sources, but currently the sampling of such nearby sources is extremely sparse, so that large uncertainties are introduced through this foreground subtraction. The SKA's RM grid will improve this situation by five orders of magnitude, allowing a complete template for the Galactic foreground to be derived and then removed (e.g., 15]).

Second, because detected RMs are lower than intrinsic RMs by a factor $(1+z)^{2}$, where $z$ is the redshift of the source in which Faraday rotation is produced, an absorber at a redshift of $\sim 3$ must have an $\mathrm{RM}$ signal $\sim 80$ times the measurement uncertainty in order to be considered significant. For many previous efforts, the uncertainties introduced by removing the Galactic foreground are as large as $\Delta \mathrm{RM} \sim 20-30 \mathrm{rad} \mathrm{m}^{-2}$, making it difficult to detect magnetic fields in distant objects unless the intrinsic RM is extremely high. With the SKA accurate RM measurements and precise foreground subtraction will allow us to reliably probe magnetic field evolution to much higher redshifts than has been previously possible.

Finally, an overriding limitation of previous studies has been the limited number of sources per redshift bin. By correlating SKA RMs with new surveys such as SDSS, a vast increase in the sample size is expected, so that different evolu- 
tionary models can be clearly distinguished.

\section{Galaxies and Protogalaxies at $z \gtrsim 2$}

At yet higher redshifts, we can take advantage of the sensitivity of the deepest SKA fields, in which we expect to detect the synchrotron emission from the youngest galaxies and protogalaxies (see contribution by Carilli, this volume). The tight radio-infrared correlation, which holds also for distant infrared-bright galaxies [7, tells us that magnetic fields with strengths similar or even larger than in nearby galaxies existed in some young objects, but the origin of these fields is unknown. The total intensity of synchrotron emission can yield approximate estimates for the magnetic field strength in these galaxies. between magnetic fields and cosmic rays. In deep observations, the SKA can detect and resolve spiral galaxies like M 51 out to redshifts $z \approx 2$, and to even larger distances if the star formation rate is higher than in M 51 (see contribution by van der Hulst, this volume). Since a dynamo needs a few rotations or about $10^{9}$ yr to build up a coherent field 4, detection of synchrotron emission in young galaxies at high $z$ put constraints on the seed field which potentially challenge dynamo models 32.

Even very distant galaxies unresolvable by the SKA may reveal polarized emission if the inclination is high or if the field structure is asymmetric, e.g. due to tidal interaction or ram pressure as has been observed in several galaxies (e.g., in NGC 2276; 25]). Interactions were much more frequent in the early Universe so excess polarization might be expected in some objects, especially if magnetic fields were already present before compression.

\section{The IGM and Cosmic Field Geometry}

Fundamental to all the issues discussed above is the search for magnetic fields in the IGM. All of "empty" space may be magnetised, either by outflows from galaxies, by relic lobes of radio galaxies, or as part of the "cosmic web" structure. Such a field has not yet been detected, but its role as the likely seed field for galax- ies and clusters, plus the prospect that the IGM field might trace and regulate structure formation in the early Universe, places considerable importance on its discovery. Once the magnetic field of the IGM is detected, a measurement of the characteristic size scales of its fluctuations can allow us to differentiate between the wide variety of mechanisms proposed for magnetic field generation in the IGM (see 272184319] for extensive reviews). While RMs of distant sources are potentially a useful probe of the IGM, to date there has been no detection of magnetic fields in the IGM; current upper limits on the strength of any such field are model dependent, but suggest $\left|B_{\mathrm{IGM}}\right| \lesssim 10^{-8}-10^{-9} \mathrm{G}[276]$.

Using the SKA, this all-pervading cosmic magnetic field can finally be identified through the RM grid proposed in $\$ 3$ Just as the correlation function of galaxies yields the power spectrum of matter, the analogous correlation function of this RM distribution can provide the magnetic power spectrum of the IGM as a function of cosmic epoch and over a wide range of spatial scales. Such measurements will allow us to develop a detailed model of the magnetic field geometry of the IGM and of the overall Universe.

As mentioned earlier, the observed RM is reduced by a factor $(1+z)^{2}$ over its intrinsic value. However, a simplistic assumption is that the comoving magnetic flux and electron density are both constant, so that electron density evolves as $(1+z)^{3}$, while the magnetic field evolves as $(1+z)^{2}$ (e.g., [43]). In this case, we expect the observed $\mathrm{RM}$ to be proportional to $(1+z)^{3}$. While this is a naive calculation, it demonstrates the general principle that RM signatures from distant objects can be quite large, even if the foreground IGM has a relatively small magnetic field.

More robust treatments are presented by Kolatt [26] and by Blasi et al. [6], who demonstrate in detail how the statistics of RM measurements as a function of redshift can be used to remove the foreground Galactic contribution, and then to extract the strength and power spectrum of magnetic fields in the IGM. These studies require a large sample of RMs from sources at known redshift, which should be obtainable by combining the SKA RM grid with the wide-field spectro- 
scopic data bases provided by SDSS and KAOS, plus with the all-sky multiband photometry of LSST and SkyMapper. For example, Kolatt 26 shows that an IGM field with strength $10^{-10} \mathrm{G}$ can be detected with 5 - $\sigma$ significance by considering extragalactic RM measurements over just a few degrees of sky, provided that the density of RM measurements is $\sim 800$ sources $\mathrm{deg}^{-2}$; the resulting correlation function is shown in Figure 5 Figure 2 of Beck \& Gaensler 3 demonstrates that this is well beyond the capabilities of current telescopes, but is easily achievable with the SKA. We note that this experiment requires sub-arcsec spatial resolution at radio wavelengths, so as to ensure accurate optical identification of polarized radio sources.

\section{Primordial Fields ?}

As emphasised elsewhere in this volume, a large part of SKA science will be unanticipated and serendipitous discoveries, which do not easily extrapolate from previous work. Thus, although speculative, we suggest here some isolated experiments which might provide some constraints on the strength and origin of magnetic fields at very early epochs.

A magnetic field already present at the recombination era might have affected the cosmogonic process [38. The constraints are summarised in Figure 6] in terms of the field's characteristic length scale.

A variety of cosmological processes can produce relatively high primordial magnetic fields, of strength $10^{-10}-10^{-9} \mathrm{G}$ at $z \sim 5$ (see [21]). Active galactic nuclei and violent star-formation activity in young galaxies may be able to generate seed fields of similar strength (e.g., 9284016, as illustrated schematically in Figure 7 The dynamo mechanism may then have amplified the seed field to the microgauss strength observed in galaxies today. RM experiments with the SKA toward very high redshift sources might provide evidence for such fields, taking advantage of the $\mathrm{RM} \propto(1+z)^{3}$ effect discussed in $₫ 7$ For example, we already know of gamma-ray bursts at redshifts as high as $z=4.5$ [1], and of radio galaxies at $z=5.2$ [41]. With the advent of projects

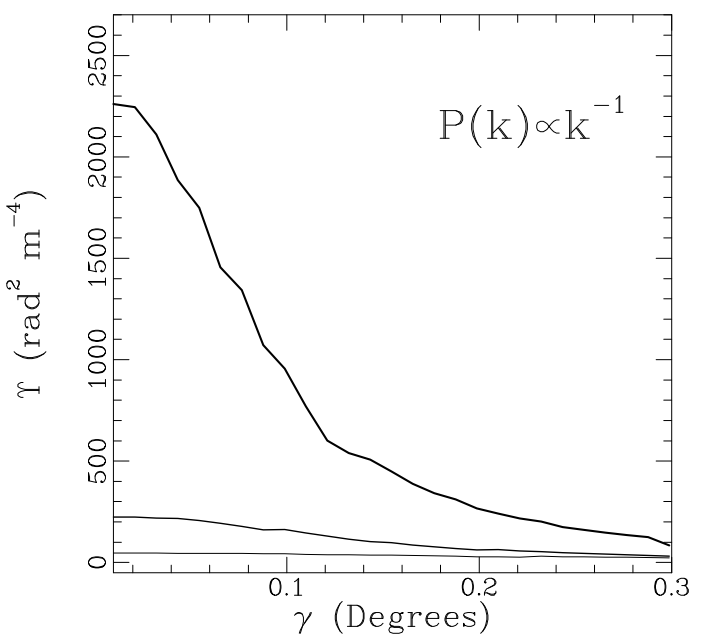

Figure 5. RM correlation between pairs of background sources as a function of separation angle, assuming a power spectrum for magnetic fluctuations of the form $P_{B}(k) \propto k$ 26. From bottom to top, the three lines show the correlation function for sources at redshifts of $z=0.5,1$ and 2 . Reproduced by permission of the AAS. 


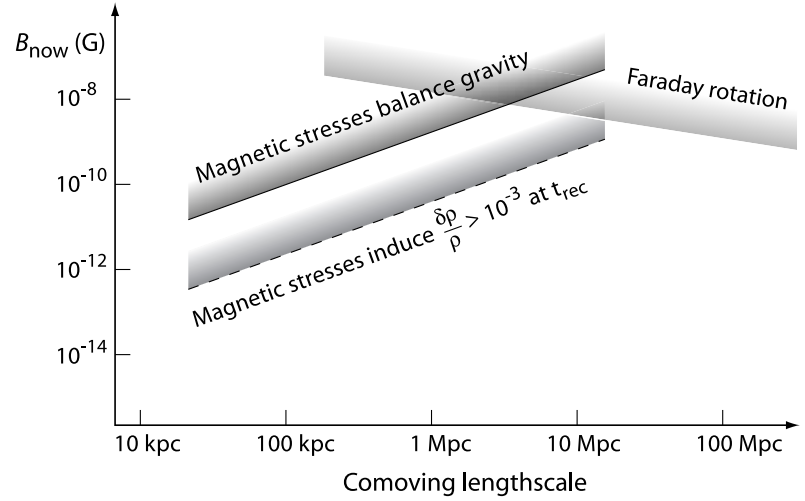

Figure 6. Observational and theoretical constraints on the magnetic field on various length scales at the time of recombination, $t_{r e c}$. A field is cosmogonically important if it can generate density perturbations of amplitude $\sim 10^{-3}$ at $t_{r e c}$, since these would have developed into gravitationally bound systems by the present time 33.

such as SWIFT and LOFAR, it is virtually certain that the sample of such high redshift sources will soon greatly expand. With the high sensitivity of the SKA, linear polarization and Faraday rotation should be detectable from some of these sources. Using a foreground template at lower redshift provided by the RM grid, the RM contribution at high redshifts can then be isolated, yielding a possible detection of magnetic fields at early epochs.

\section{Conclusion}

We have outlined the exciting new insights which the SKA can provide into the origin, evolution and structure of cosmic magnetic fields. The sheer weight of RM statistics which the SKA can accumulate, combined with deep polarimetric observations of individual sources, will allow us to characterise the geometry and evolution of magnetic fields in galaxies, in clusters and in the IGM from high redshifts through to the present. We may also be able to provide the first constraints on when and how the first magnetic fields in the

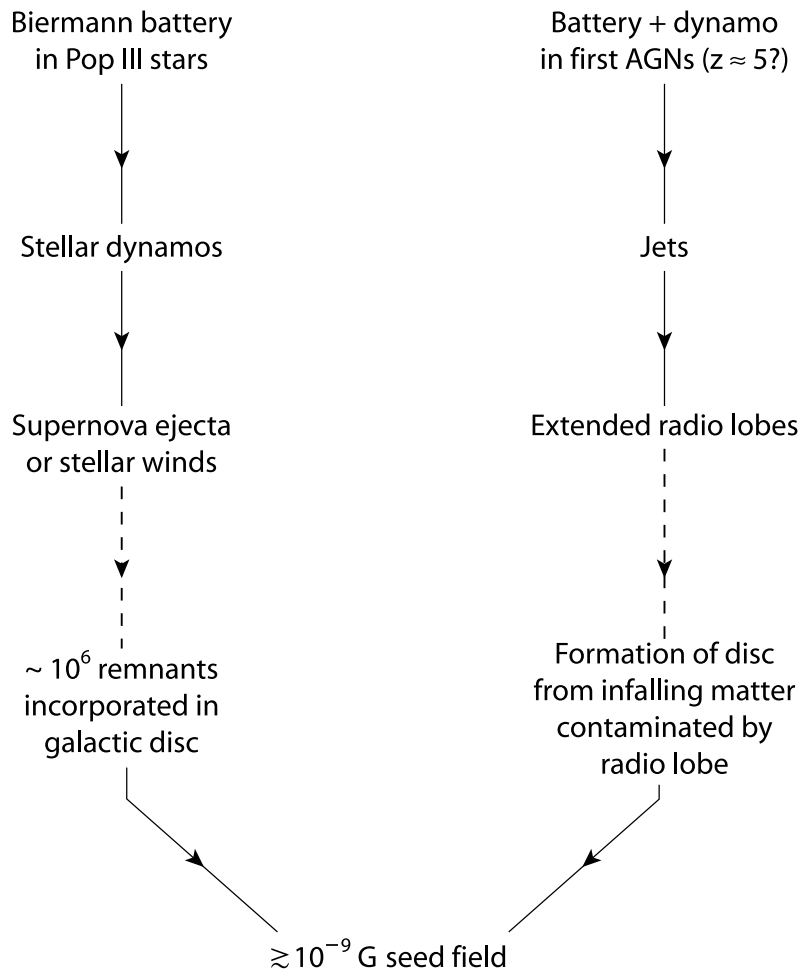

Figure 7. Suggested mechanisms through which Population III stars and active galactic nuclei can both produce seed magnetic fields of strength $\gtrsim$ $10^{-9} \mathrm{G} 34$. 
Universe were generated. Apart from these experiments which we can conceive today, we also expect that the SKA will certainly discover new magnetic phenomena beyond what we can currently predict or even imagine.

The main SKA specifications which enable us to reach our goals are high polarization purity, spectropolarimetric capability, and a frequency coverage of at least $0.5-10 \mathrm{GHz}$. The AllSky RM Survey is best done at a frequency of $1.4 \mathrm{GHz}$, perhaps simultaneously with other widefield continuum and $\mathrm{H}$ I surveys. Faraday tomography requires frequencies $\sim 0.5-1 \mathrm{GHz}$ for low-density, and $\sim 1-5 \mathrm{GHz}$ for high-density regions of the local ISM. Mapping of the polarized synchrotron emission in the Milky Way, external galaxies and clusters is best done at frequencies $\gtrsim 5-10 \mathrm{GHz}$ where Faraday depolarization is minimal. Other minimum requirements are a field of view at $1.4 \mathrm{GHz}$ of $1 \mathrm{deg}^{2}$ which can be fully imaged at $1^{\prime \prime}$ resolution, a significant $(\sim 50 \%)$ concentration of the collecting area into a central core of diameter $\sim 5 \mathrm{~km}$, and a largest baseline of $\sim 300 \mathrm{~km}$.

To summarise, incredibly rich data sets for the study of magnetic fields await the advent of the SKA. With the unique sensitivity, resolution and polarimetric capabilities of this next-generation radio telescope, we can finally address fundamental questions relating to the physics of cosmic magnetic fields, and their role in forming structure on all scales.

We thank JinLin Han, Martin Rees, Wolfgang Reich, Anvar Shukurov, Dmitry Sokoloff, Kandu Subramanian, Marek Urbanik, Ira Wasserman and Ellen Zweibel for useful comments. B.M.G. acknowledges the support of the National Science Foundation through grant AST-0307358. The National Radio Astronomy Observatory is a facility of the National Science Foundation operated under cooperative agreement by Associated Universities, Inc. The Australia Telescope is funded by the Commonwealth of Australia for operation as a National Facility managed by CSIRO.

\section{REFERENCES}

1. Andersen, M. I. et al., 2000, A\&A, 364, L54

2. Beck, R., 2000, Phil. Trans. R. Soc. Lond. A, 358,777

3. Beck, R., Gaensler, B. M., this volume

4. Beck, R., Poezd, A.D., Shukurov, A., Sokoloff, D., 1994, A\&A, 289, 94

5. Beck, R., Brandenburg, A., Moss, D., Shukurov, A., Sokoloff, D., 1996, ARA\&A, 34, 155

6. Blasi, P., Burles, S., Olinto, A. V., 1999, ApJ, 514, L79

7. Carilli, C.L., Bertoldi, F., Omont, A., Cox, P., McMahon, R. G., Isaak, K. G., 2001, AJ, 122, 1679

8. Carilli, C.L., Taylor, G.B., 2002, ARA\&A, 40, 319

9. Chakrabarti, S.K., Rosner, R., Vainshtein, S. I., 1994, Nature, 368, 434

10. Chandran, B.D. G., Cowley, S.C., 1998, Phys. Rev. Lett., 80, 3077

11. Clarke, T. E., Kronberg, P. P., Böhringer, H., 2001, ApJ, 547, L111

12. Feretti, L., Johnston-Hollitt, M., this volume

13. Fletcher, A., Beck, R., et al., 2004, in preparation

14. Fomalont, E. B., Ebneter, K. A., van Bruegel, W. J. M., Ekers, R. D., 1989, ApJ, 346, L17

15. Frick, P., Stepanov, R., Shukurov, A., Sokoloff, D., 2001, MNRAS, 325, 649

16. Furlanetto, S. R., Loeb, A., 2001, ApJ, 556, 619

17. Gaensler, B. M., Dickey, J.M., McClureGriffiths, N. M., Green, A. J., Wieringa, M. H., Haynes, R. F., 2001, ApJ, 549, 959

18. Gaensler, B. M., Manchester, R. N., Green, A. J., 1998, MNRAS, 296, 813

19. Giovannini, M., 2003, preprint (astro-ph/0312614)

20. Govoni, F., Taylor, G. B., Dallacassa, D., Feretti, L., Giovannini, G., 2001, A\&A, 379, 807

21. Grasso, D., Rubinstein, H.R., 2001, Phys. Rep., 348, 163

22. Han, J. L., Beck, R., Berkhuijsen, E. M., 1998, A\&A, 335, 1117

23. Haverkorn, M., Katgert, P., de Bruyn, A. G., 2003, A\&A, 403, 1031 
24. Hopkins, A. M., Afonso, J., Chan, B., Cram, L.E., Georgakakis, A., Mobasher, B., 2003, AJ, 125,465

25. Hummel, E., Beck, R., 1995, A\&A, 303, 691

26. Kolatt, T., 1998, ApJ, 495, 564

27. Kronberg, P. P., 1994, Rep. Prog. Phys., 57, 325

28. Kronberg, P. P., Lesch, H., Hopp, U., 1999, ApJ, 511, 56

29. Kronberg, P.P., Perry, J. J., Zukowski, E. L. H., 1992, ApJ, 387, 528

30. Narayan, R., Medvedev, M. V., 2001, ApJ, 562, L129

31. Oren, A. L., Wolfe, A. M., 1995, ApJ, 445, 624

32. Perry, J. J., Watson, A. M., Kronberg, P. P., 1993, ApJ, 406, 407

33. Rees, M., New Perspectives in Astrophysical Cosmology, 2nd edition, 2000, Cambridge Univ. Press

34. Rees, M., in Magnetic Fields in the Universe, ed. R Wielebinski, 2004, Springer, Berlin

35. Schulman, E., Fomalont, E. B., 1992, AJ, 103, 1138

36. Sokoloff, D. D., Bykov, A. A., Shukurov, A., Berkhuijsen, E. M., Beck, R., Poezd, A. D., 1998, MNRAS, 299, 189

37. Stepanov, R., Frick, P., Shukurov, A., Sokoloff, D., 2002, A\&A, 391, 361

38. Subramanian, K., Barrow, J. D., 1998, Phys. Rev. Lett., 81, 3575

39. Uyanıker, B., Landecker, T.L., Gray A.D., Kothes, R., 2003, ApJ, 585, 785

40. Völk, H. J., Atoyan, A. M., 2000, ApJ, 541, 88

41. van Breugel, W., De Breuck, C., Stanford, S. A., Stern, D., Röttgering, H., Miley, G., 1999, ApJL, 518, L61

42. Welter, G. L., Perry, J. J., Kronberg, P.P., 1984, ApJ, 279, 19

43. Widrow, L. M., 2002, Rev. Mod. Phys., 74, 775

44. Wolfe, A. M., Lanzetta, K.M., Oren, A. L., 1992, ApJ, 388, 17 